\title{
OBSERVATIONS ON THE EFFECTS OF SKILL TRANSFER THROUGH EXPERIENCE SHARING AND IN-PERSON COMMUNICATION
}

\author{
Vestad, Håvard; Kriesi, Carlo; Slåttsveen, Kristoffer; Steinert, Martin \\ Norwegian University of Science and Technology
}

\begin{abstract}
An essential part of any space in which physical prototyping and prototype-driven product development is being conducted is the education of its users in the necessary skills to fully utilise the material resources of the space. This paper describes how two different skills were transferred between five projects in our research laboratory, TrollLABS. Based on the observed skill-transfers in the production of PCBs and use of RF-communication in mechatronics projects certain tendencies emerged: How the use of forced vocal experience sharing; And in-person transferring of skills has impacted the acquired skills of the learner. The observations further show that through the guidance of a more experienced user the learner is able to make "skill-jumps": Intermediate skill steps, as well as underlying detailed knowledge, are skipped and the learner is able to reach a high skill level in a shorter time than the original acquirer of the skill. Furthermore, skills are retained in the space through cross-generational collaboration and communication. This article aims to share these insights and provide a starting point for answering some of the challenges of modern maker spaces.
\end{abstract}

Keywords: New product development, Case study, Training, Makerspace, Prototyping

Contact:

Vestad, Håvard Nitter

Norwegian University of Science and Technology

MTP

Norway

havard.vestad@ntnu.no

Cite this article: Vestad, H., Kriesi, C., Slåttsveen, K., Steinert, M. (2019) 'Observations on the Effects of Skill Transfer through Experience Sharing and In-Person Communication', in Proceedings of the 22nd International Conference on Engineering Design (ICED19), Delft, The Netherlands, 5-8 August 2019. DOI:10.1017/dsi.2019.23 


\section{INTRODUCTION}

Prototyping is considered an essential element of the product development process (Eppinger and Ulrich, 2011) and multiple methods, such as Wayfaring (Steinert and Leifer, 2012) and Design Thinking, rely on rapid iterations in order to learn from the prototypes (Leifer and Steinert, 2011). If used correctly, prototypes enable finding unknown unknowns (Kriesi et al., 2016) and can lead to rapid development of unforeseen solutions in different contexts, like biomedical engineering (Nygaard et al., 2018), heavy industries (Winjum et al., 2017), and mass-manufacturing (Kriesi et al., 2018).

Creating prototypes, however, requires resources on multiple levels, such as tools, machinery, materials, and associated skills. Makerspaces are meant as a physical location where these elements are combined, and prototyping is subsequently facilitated. This view is shared by industrial (Jensen et al., 2016) and educational stakeholders (Chou, 2018; Forest et al., 2014), as their investments in establishing a "makerspace" within their entities show.

While hardware, such as machines, tools, and materials, are easy to acquire, skills are linked to people and their experiences and are therefore hard to transfer, retain, and share in a structured way. As highlighted by Jensen et al. (2016), one of the main challenges regarding the successful design of a makerspace is "transforming novel users into experienced ones". In our prototyping laboratory, TrollLABS, this challenge has been met by incorporating weekly stand up meetings where the users share their experiences from their weekly sprint (Slåttsveen et al., 2018). Additionally, projects are worked on side by side in the same space; with little incentive for internal competition this might further encourage sharing of skills, resources and experience. These observations from within our prototyping laboratory triggered a discussion on skill sharing and experience exchange within the research group. Based on the observations of the cases presented in this article, we propose a ground work for further experimental investigations on how skills are shared, and which effect sharing of skills has on project productivity in an open makerspace-like lab.

\subsection{The early stage}

Prototyping within the early stage of product development, the fuzzy front end (FFE), means iterating, testing, and adjusting the design in order to find a great solution. One method that we teach is the wayfaring model (Steinert and Leifer, 2012). It relies on targeted design probes in order to make the next step within the solution space. However, given the complexity of most challenges, this also means that the teams or individuals working on a challenge need to iterate within multiple knowledge domains, meaning they need to use a multidisciplinary approach to the problem. Figure 1 depicts the wayfaring model. While a multidisciplinary approach increases the potential amount of solutions, it also creates the challenge of potentially lacking in-depth knowledge of certain disciplines. Within TrollLABS alone - located at the department of mechanical engineering - our projects have already required in-depth knowledge of sociology, artistry, psychology, medicine, computer science, and mechanics. As such, the products also require an overlapping, multidisciplinary range of skills that need to be mastered to carry out their development. This makes the question of skill retention a fundamental challenge, that is frequently addressed in our research lab.
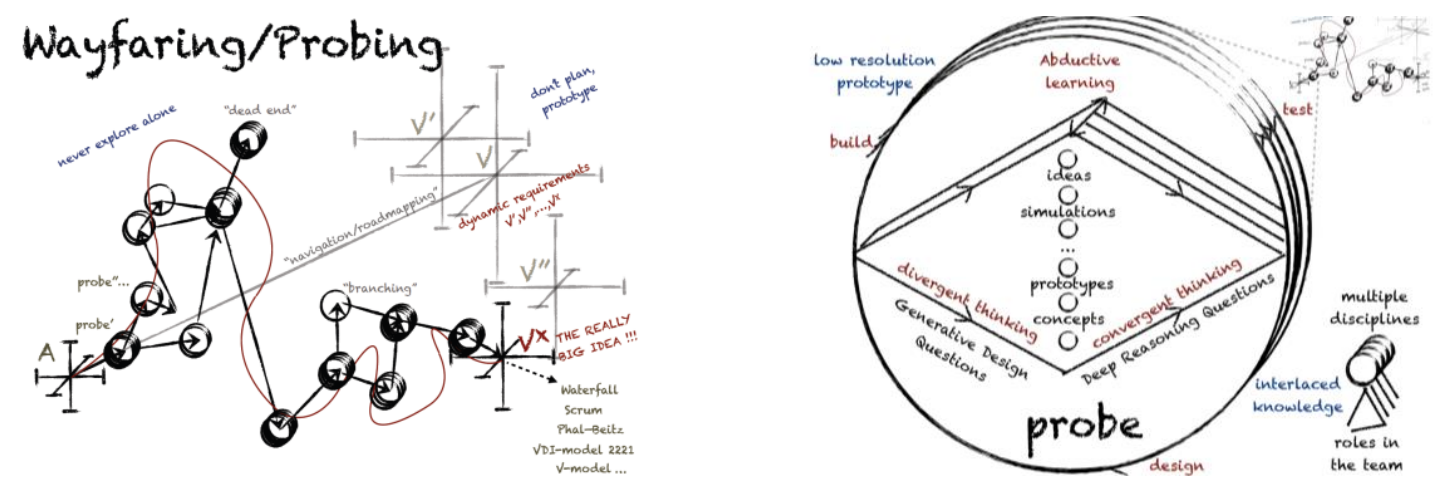

Figure 1. The wayfaring principle: Each circle along the path (left) represents a multidisciplinary probe (right) consisting of design-build-test circles. With permission from Gerstenberg et al. (2015) 


\section{METHOD}

TrollLABS is in its core similar to the concept of makerspaces (Jensen et al., 2016), as it is meant to facilitate the making and testing of open-ended ideas and prototypes (Forest et al., 2014). However, as a research laboratory TrollLABS offers a high degree of control when compared to its traditional makerspace counterparts: At any given time, the researchers at the labs have control over who has access, which machines and materials are in use, and which projects are currently being conducted. Like other makerspaces, one of the challenges is skill retention within the lab: Researchers and students will both frequently finish their research projects and take their skills elsewhere, e.g. the industry. In order to get an idea of how skills are currently being transferred in our makerspace, and to form some ideas for further research the authors have observed five projects that were conducted within the lab, where the authors were either directly or indirectly involved.

Among the five projects two cases of skills transfers were deemed of interest: The first case concerns transfer of skills when it comes to the production of printed circuit boards (PCB) and includes three projects: eduROV, CellFlow, and TrollBOT. In each project an author was main contributor. The second case concerns the use of radio frequency (RF) communication in mechatronics prototyping and consists of: TrollBOT, a "water snake robot", and "medical manikin eyes". In the first project one author was one of three main contributors, whereas in the latter two an author each were involved as supervisor for one and three students, respectively.

\subsection{Aim and objective}

This article aims to promote research questions that emerge from the observations and dissemination of the phenomena observed in the cases as presented in section 3 . The close relationship to the projects allowed the authors to make well-informed observations about the projects from an internal perspective and using them as cases in order to investigate similarities between them (Eisenhardt, 1989; Yin, 2013). By examining these cases and their similar features one can deduct research questions and hypotheses. The objective of this work, and such a study, is therefore "not to conclude a study but to develop ideas for further study." (Yin, 2013, p. 179). The emerging research questions are presented in section 5 .

\subsection{Skills vs. knowledge}

Within this article the terms "skill" and "knowledge" must be defined for clarification: "Skill" relates to an individual's ability to do something. "Knowledge", however, refers to an individual's ability to explain the required theoretical foundation of a principle. One way of understanding the extreme interpretation of this article's distinction is: skills are acquired in the workshop and knowledge is acquired in lecture halls. Without making a judgement on whether skill or knowledge is more important, one has to be aware that there is a difference.

\section{CASES}

\subsection{Case 1: PCB production}

PCBs enable highly compact design of electronic components by replacing the wires with very thin traces of copper on a plastic plate. Consequently, they are a central element in all electronical devices. When prototyping mechatronics, breadboards are often the first step to verify a circuit that you have designed. Breadboards allows for components to be placed and electrically connected without permanently bonding them. The next advancement of the circuitry, depending on its complexity, would be soldering and therefore fixating wires and components. Moving to PCBs requires skills with machinery and design software that might be less straight forward than the previous methods and thus previous prototypes would often not reach this level of fidelity.

While there are many more skills that are used in a variety of products, e.g. CAD design, the PCB example is chosen since the skill for producing them was acquired from scratch within our group, particularly due to the interest of one individual $\mathrm{PhD}$ candidate. It has since diffused in rapid manner into various student teams. Furthermore, the three projects, eduROV, CellFlow, and TrollBOT, were independently developed, highlighting the importance of close collaboration, exchange, and unobstructed sharing of skills. Figure 2 qualitatively shows the timeline of the skill diffusion process across these three projects. 


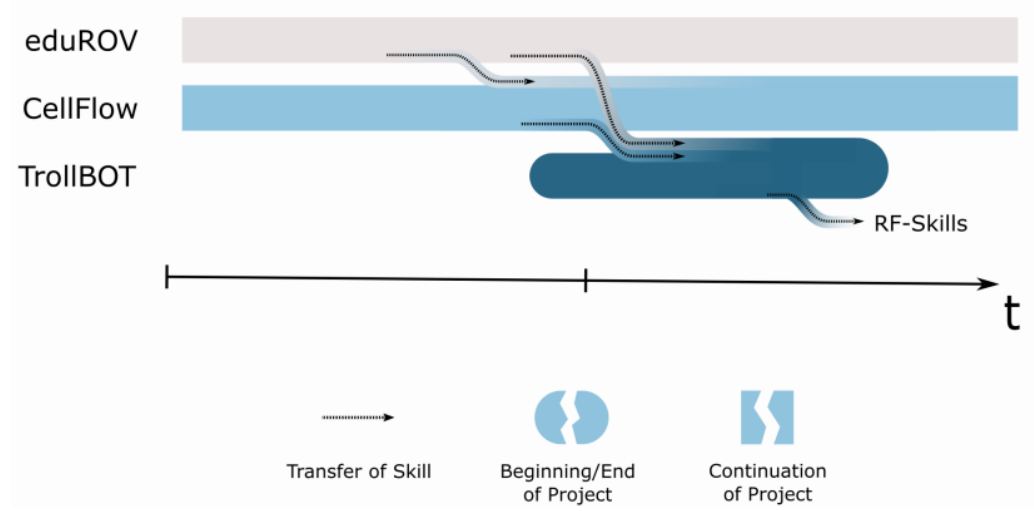

Figure 2. A relative timeline of projects involved in Case 1, showing the overlap of the project periods. Arrows highlight the flow of skills transferred between the projects.

\subsubsection{EduROV}

After being inspired by an initiative from a local Maker Faire, one of the PhD-students of the lab decided to develop an open-source low-cost ROV (remote-controlled submarine) as a practical educational project. The ROV is designed to be built by high-school students or hobbyists and it should provide a learning experience. The technology required to make such a product ranges from computer science, through design of electrical systems, mechanical design and construction. The final design is made up of an external frame, a waterproof hull containing a Raspberry Pi and an Arduino microcontroller mounted on a custom-made PCB making up the motor-control circuitry. The vehicle is controlled from a laptop through an ethernet cable acting as the ROV's umbilical cord.

Particularly the development of the PCB required the designer to obtain a new set of skills. The designer had a good understanding of the electrical components and circuitry, but the CAD tools required to make the production files for an outsourced production of the prototypes required in-depth learning. Taking advantage of local resources from TrollLABS, a CNC-mill was used to produce twosided circuit boards in-house until the design was fixed. Subsequently the designer ordered professionally produced PCB's, using the same production files as used in the CNC-mill. Iterations of the PCB-design, production complexity and fidelity level are shown in Figure 3.

With little previous experience within the lab on PCB design and production, the project started from scratch and the developer had to acquire the skills to produce and design the PCBs from external sources. Likewise, he had to find the most suitable method to do so with either the equipment already in the lab or acquiring new equipment. The designer had to source machinery, tools, and software, making it a time intensive part of the project.

\subsubsection{Project CellFlow}

In collaboration with a cell research group another PhD-student developed a device that allows for exposing cells to more in vivo like conditions, while staying in vitro. Even though a lot of the developments circled around miniaturisation of heaters and other mechanical challenges, the internal temperature control, as well as the user interface rely on an electric circuit with sensors, display, buttons, and microcontroller. Initial versions of the circuitry were simply to test other fundamental principles, e.g. how to control the heater, and thus the size did not matter. Over time, however, the electronics needed to find space within the system, which required downsizing and switching to PCBs. The changing nature of the components made it necessary to be able to rapidly change the layout of the circuitry. The development and applied means of manufacturing the PCBs correlate with the learning process of the according skills for both, software and production machines. The four main iterative steps of the development are shown in Figure 4. 


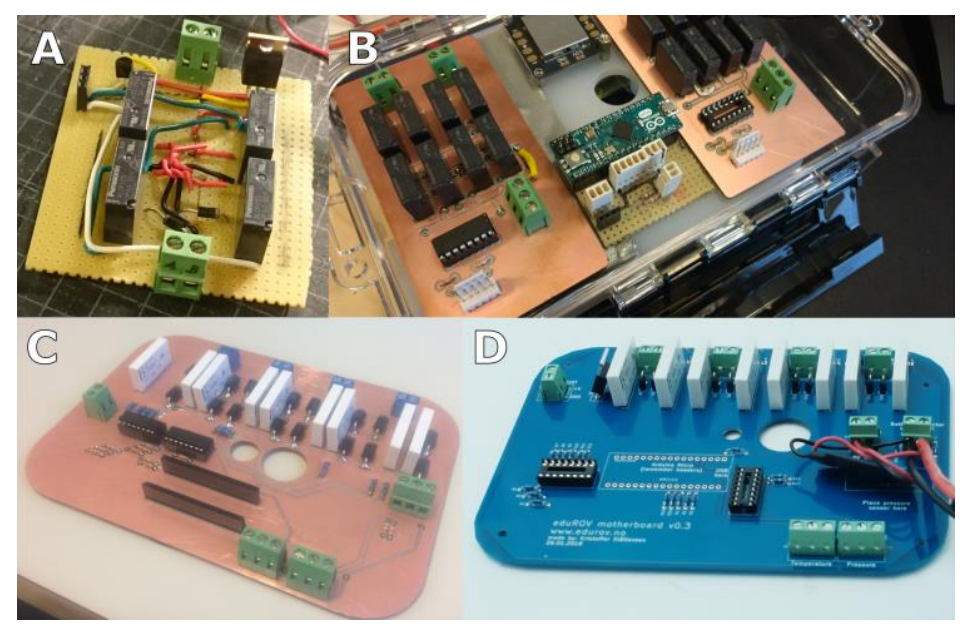

Figure 3. Four iteration steps in developing the motor-controller PCB for the eduROV, listed from low to high fidelity: $(A)$ Concept prototype; $(B)$ First functional prototype; $(C)$ Pre-production prototype; (D) First external production version and current design state.

Case 1

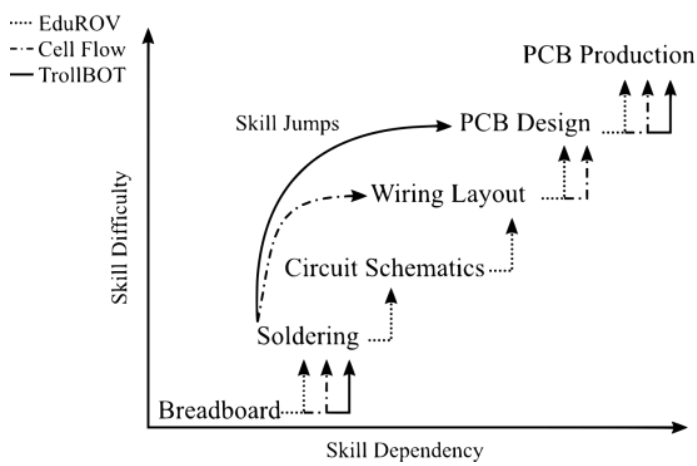

Case 2

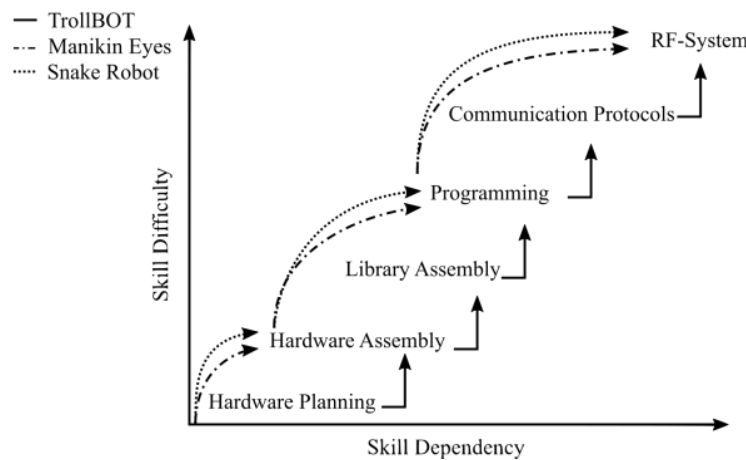

Figure 4. Simplified and qualitative skill development throughout the two cases, highlighting the shortcuts that skill sharing enabled

\subsubsection{TrollBOT}

While the previous two projects started from low-resolution PCB prototypes, a skill-jump can be seen by a project-team in our one semester, 7.5 ECTS, course 'TMM4245 Fuzzy Front End'. The team was working on a cube-based robot platform to easily be interchange functionalities in robotics prototyping, the cube-concept can be seen in Figure 5. The communication between each module was RF-based and required good and stable connections in the circuitry. Since each cube needed the same circuit, using machinery to produce the circuits was quickly desired. They caught up with the owner of the eduROV and CellFlow projects through a weekly meeting and started producing two-sided PCBs right away. Being a one semester project the time from idea to final prototype was very short. With much of the time spent on need finding and exploration of the solution space, only about two months were spent on designing and prototyping the modular robot system. The ability to utilise previous knowledge in PCB design quickly gave the team a relatively high technical production ability which enabled the team to play around with and try other things, thus the team was also able to learn how to make Arduino libraries for RF communication with simple Arduino enabled RF chips. The time saved could be used elsewhere. The confidence level within the lab for PCB production enabled the team to not only use the CNC to produce circuits for verification pre-production, but also to prototype the circuit layout and components.

\subsection{CASE 2: RF communication}

RF signals are frequently used to remotely control electronic products and enables communication over potentially long distances. While the technology has existed for a long time, implementing wireless 
communication in simple prototypes can often be time consuming, as the freedom and flexibility to send any format of messages means that protocols should be established to send and read data packages sent between modules. With the growth of IoT enabled devices, multiple simple solutions have reached the market that enables production of simple RF devices, such as LightBlue Bean, ZUNO, XBee, NodeMCU, etc. User friendliness is often interlinked with higher price point while the simpler, cheaper, RF chips often lack good support. The price, either in purchase, work, or knowing how to balance the two, meant that few projects were made at our lab that utilized this technology. A project that tried to solve these challenges was the TrollBOT project.

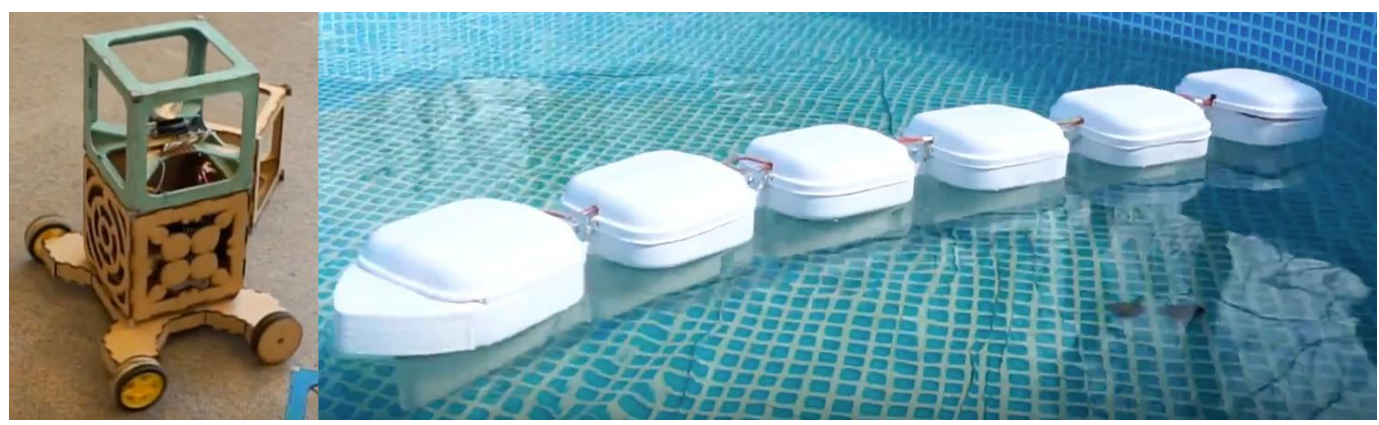

Figure 5. The TrollBOT (left) that shares technology with the water snake (right).

\subsubsection{TrOIIBOT}

One of the challenges during the TrollBOT project was finding flexible ways to run signal and power wires to interchangeable units in a simple and modular way. The solution became RF-signals. To enable multiple nodes, the team opted to use cheap open source Arduino-enabled boards, Arduino micro, and RF chips, specifically the nrf24101 model (Nordic Semiconductors, Trondheim, NO). This meant that the team had to invest work into enabling communication between the modules. To ease the use of RFsignals in the writing of code, a library was made where one master could control multiple nodes. The nodes were appointed names so that code that was meant to run on the node module could be written on the master as normal Arduino code, but addressed with the nodes name before. In addition, the library package included the necessary sub libraries needed for the Arduino to speak with the RF-chip. To do so the team had to learn how the existing libraries that communicated with the RF-chip handled code and messages. They then had to read up on protocols and coding to make a new library around these that enabled the simplified coding style, both at the master- and node end.

\subsubsection{Water snake robot}

In the same FFE class as the TrollBOT project team, another team were building a swimming snake robot as seen in Figure 5. The robot was controlled remotely and produced propulsion through snake like movements controlled by an Arduino. While the group was quickly able to produce the swimming patterns necessary for forward propulsion and control of the robot based on their previous experience, they reached a limitation to this when confronted with wireless control of the robot. When faced with the problem of sending signals to the robot they connected with the TrollBOT project through a weekly meeting to adopt the library to quickly add RF control and communication to their project.

\subsubsection{Medical manikin eyes}

Simultaneously with the FFE course work, the teaching assistant of the FFE- course was working on his master's thesis where he was constructing mechanical eyes for medical manikins (Nygaard et al., 2018). The eyes offer a central patient-doctor interaction point and realistic motions are essential in a training environment. In order to remove the operator of the actuated manikin eyes from the manikin itself, the designer wanted to use wireless communication. Instead of trying to find external resources and documentation on how to establish a wireless communication system between the operator and the eyes, the designer chose to instead confront the TrollBOT group to learn how to use the library they had made. The library and accompanying programming skills were in this way picked up by projects also outside of the course. While the spiking interest in using the library lead to the program and documentation being shared online, these initial transfers of the skill was done through personal 
exchanges and explanation of the library. The projects from Case 2, ended at approximately the same time, but the RF skills learned were further used in new projects. Most notably they were included in as resources in another course "TMM4150 Machine Design and Mechatronics" taught by one of the authors the following semester.

Modules such as the RF-chip (nRF24101) used in this example are usually accompanied by libraries and examples on how to use them. When working with multiple different modules, the multitude of code calls and syntaxes can become tedious, confusing and result in code that is hard to follow. The library constructed was simple in its composition and offered little advancement in the way messages where sent between modules as compared to existing libraries and examples for the RF chip. The library simply focused on easing the users interfacing with how to code the interaction between the modules in an intuitive way by using the syntax of the Arduino IDE that the users of our lab were mostly familiar with which meant that it was fast to use and learn. The library was quickly picked up by the mentioned projects, even before the library was finished, showing how a new skill when needed by other projects can quickly diffuse to other projects when communicated verbally, as shown in Figure 6.

Timeline of Skilltransfers in Case 2

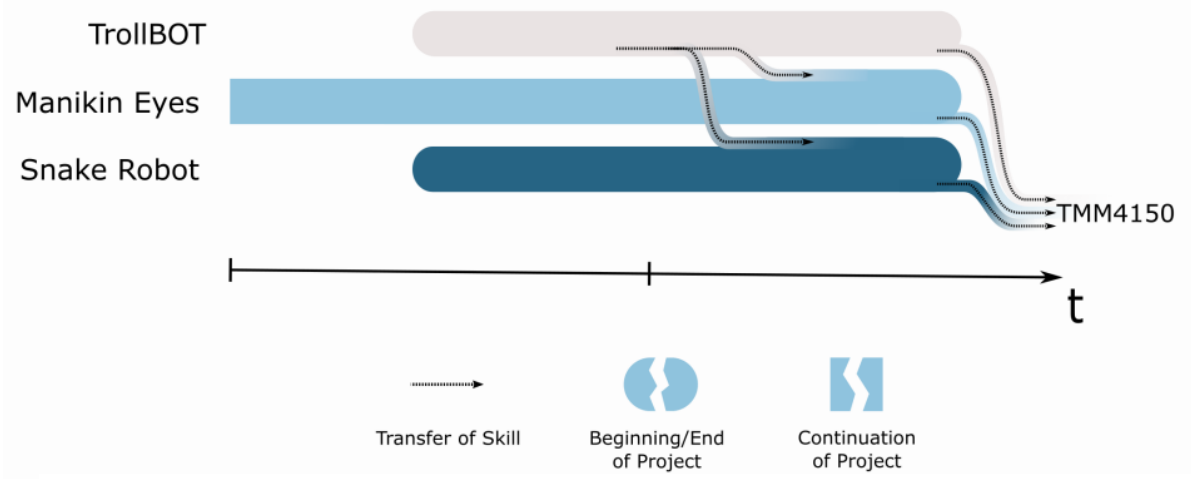

Figure 6. A relative timeline of projects involved in Case 2, showing the overlap of the project periods. Arrows highlight the flow of skills transferred between the projects.

\section{ON THE TRANSFER OF SKILLS}

Skills are commonly transferred and retained by creating extensive documentation. With easy videohosting and other, more interactive ways of storing information, it surely has become easier to create documentations. Nevertheless, complete, fool-proof instruction file takes a large amount of time to create and follow up on and can be subject to many pitfalls: instructions need to make some assumptions about the skill level and familiarity of the reader/observer within the learning field. When such assumptions are not correct or properly conveyed it can become a loop of misunderstandings (Nonaka et al., 2000). Furthermore, software updates or a change of machinery can render any documentation outdated and irrelevant. Novel users that only need one specific partial skill to progress in their development phase do not necessarily want to work themselves through all the available information for the complete skillset. A single piece of advice from a skilful user will often be preferable.

Rather, the 'learning by doing' - a master-apprentice approach can be utilised: by watching skills being applied by an experienced master user, the apprentice can pick up on key elements and apply them, independent of the level of understanding of the rest of the potential applications. In addition, the apprentice understands the nature of the process, rather than simply the step-by-step instructions of a specific tutorial.

However, transferring of skills is not divisible into only two schools of thought. Rather a multitude of theories between and outside of the mentioned approaches exist. As an example, a middle ground between the two methods, where the new skill is applied simultaneous with the instructions has been shown to give a much higher learning outcome for the novice for some skills, as compared to pure instructions in massive open online courses (MOOC) (Koedinger et al., 2015). 
From the cases presented, skills transferred in TrollLABS preferred a verbal and personal approach, either actively through weekly meetings or passively through co-location communication. It is not necessarily clear if other methods could have produced more preferable learning outcomes.

\subsection{Facilitating prototyping and keeping experiences}

The multidisciplinary solutions that often occur in the projects at TrollLABS result in a multitude of skills that are accumulated within the users of the lab. However, there is one big problem: Since we are a research laboratory at a university, the duration of the stay of the most experienced users $-\mathrm{PhD}$ students - is limited. Consequently, any accumulated knowledge and skills will get lost, unless they are somehow transferred to younger users. Clearly, new users can acquire the same skills again from scratch, but this will most likely come at a high price for these new projects in terms of time spent "reinventing the wheel". This understanding is supported by e.g. Forest et al. (2014) who used 10 skilful supervisors to ensure skill transfer within their makerspace. They further make a point out of staffing their supervisors separate from the course to ensure skill transfer between the user generations.

With a similar realization we often question how one can combine a rapid, agile product development process with targeted skill diffusion, since ideally every product development team within the lab should have all the skills needed to get the maximum out of the available machinery. A way to force cross communication between projects and sharing of skills and knowledge that we have adopted is the use of weekly stand up meetings, similar to those found in SCRUM-sprints. Here, both, master- and PhD students (10-20 people) share their project related progress, issues, and goals (Rising and Janoff, 2000). Slåttsveen et al. (2018) has studied some of the perceived effects of these meetings when applied in the introductory FFE course. While the pressure of presenting progress every week might encourage a higher level of production, the communal discussion around the problems facing the project can also be of high value. All the users of the lab can give feedback and tips on how to solve their challenges and which contributions in form of skills, knowledge, experience or other resources the users can find within the lab network. Regarding the cases, keeping an open communication with other projects enabled the projects to use specific skills from the skillset acquired by the first learners. In projects, time is a limited resource and by using already acquired resources within the lab the threshold for undertaking a challenge is lowered. For new skills this is especially true; you know that someone with similar background as your own have been able to grasp the skill, and you also know that you have the support and help to learn the skill should you need it. As Kelley \& Kelley (2013) write, often there is just a little reassurance needed in order to get people comfortable with applying their skill.

\section{DISCUSSION}

Makerspaces have a common goal that is in the name: To make something. However, machines and tools do not make anything on their own - they need input from users. Furthermore, the users of a space change, while the machines stay in place. Addressing these challenges of how to create more experienced users and how to retain skills within a group, we presented two cases that both show how new skills were acquired and further used and implemented by other users of the lab.

In the cases of both the PCB- and RF skill transfer, an initial learner put in the time and effort to investigate the required knowledge and theoretical background to gain a new skillset. Other users of the lab were then able to acquire the necessary skills to complete their own projects from the initial learner through peer-to-peer-like guidance initiated by experience sharing in forced stand up meetings as well as project work being conducted in close proximity to one another.

Before presenting the research questions one should note that the closed involvement of the authors in all of the projects makes observations and perspectives susceptible to potential biases from the authors. However, the close interaction also ensured in-depth understanding of the progress and learnings in the cases.

\subsection{Effects of skill sharing}

In the cases presented the projects are making use of the same fundamental knowledge yet start using it at different skill levels. The cases show how teams can progress quicker when they do not need to gather extensive knowledge but can rather branch off from a higher skill level. These observed skill jumps are illustrated in Figure 4, for both cases. This observation is in analogy with the different stages of skill acquisition as described by Benner (1994). This is exemplified by how little time was invested in 
learning how to make PCBs so that the TrollBOT project was able to acquire new skills in RF communication, which in turn moved the starting conditions for other projects in the lab. As a result, the subsequent projects that based their prototypes on skills that were already mastered by other users in the lab, were able to make prototypes of similar fidelity as those before much faster, and often reaching a higher end fidelity by focusing work elsewhere. By using skills from the neighbours tool-set, the prototyping activities were accelerated.

When dealing with the sharing and retention of skills one is dealing with humans and their interactions and communication. Consequently, it is difficult to determine a clear set of actions to retain skills in makerspaces, as it itself is a multidimensional problem influenced both by physical, social, and psychological factors. Through the projects presented in the two cases the observations show that sharing experience between users can be an efficient way to retain skills within the lab by distributing the acquired skill to more users and projects cross generation. Such as the PCB creation skills that came through work on a $\mathrm{PhD}$ thesis but was absorbed by younger students in their course work, and the RFlibrary that diffused from graduation course coursework to work in a master's thesis.

As multidimensional terms, skills, skill level and prototype fidelity are hard to quantify, and through the presented cases we have not made an effort to do so. We have rather focused on noting how we perceived the relative changes effecting the projects.

The first emerging research question regarding the effects of skill sharing is: "Does skill sharing accelerate progress within innovation communities?"

\subsection{How skills are shared}

We observed that skills were mostly shared in-person either actively through forced weekly meetings or passively through cross communication due to co-location. The premises of the work done within TrollLABS might differ from other settings where product development activities are being conducted. Being a research lab there is no true economic incentive to outperform other projects and teams, sharing both skills and knowledge is not a loss of advantage but rather an investment into the lab itself. As such, the trends that we have observed through these cases may not hold true in other maker spaces. It is possible that our observations are just an anomaly within our research group, and that our users are more open to this sort of skill sharing than others. We were not able to distinguish any differences in the effects or efficiency between the active and passive form of in person skill transfer as they quickly go through the same steps for the transfer, with only the initiation differentiating them. Neither could we compare the personal skill transfer to the classical documentation-based approach, as the documentation approach was only fully utilized by the initial skill-learners in the eduROV and TrollBOT project.

The second emerging research question regarding how skills are shared is: "Does active, passive, and documentation-based skill-sharing affect skill retention, and if so: What is the underlying mechanism?"

\subsection{Further work}

To find the underlying mechanisms behind the research questions we propose to conduct fitting experiments. One can imagine similar setups as described by Dow et al. $(2009,2012)$ where participants engage in a design activity with a measurable, quantifiable outcome. Subsequently, one can adjust an independent variable to observe the effect on the dependent ones.

Alternatively, certain skill sets can be tracked in higher detail throughout projects conducted within a laboratory setting in order to form a stronger data foundation in future studies. A closer observation of skill dissemination and application could bring forward a very strong argument for, or against, the proposed skill sharing.

Although the amount of data presented in this article is limited, we have observed throughout our projects that sharing skills frequently has helped accelerate project progress. Therefore, we would encourage makerspaces, as an alternative to extensive documentation, to assemble their users to share stories of success and failure and follow up with sharing required skills.

\section{REFERENCES}

Benner, P. (1994), "From novice to expert", in: A Collection of Readings Related to Competency Based Training. Deakin University, Victoria, Australia, pp. 129-137. 
Chou, P.-N. (2018), "Skill development and knowledge acquisition cultivated by maker education: Evidence from Arduino-based educational robotics". EURASIA J Math Sci Technol Educ Vol. 14, pp. 1-9. https://doi.org/10.29333/ejmste/93483

Dow, S.P., Glassco, A., Kass, J., Schwarz, M., Schwartz, D.L. and Klemmer, S.R. (2012), "Parallel Prototyping Leads to Better Design Results, More Divergence, and Increased Self-efficacy", in: Design Thinking Research, Understanding Innovation. Springer, Berlin, Heidelberg, pp. 127-153. https://doi.org/10.1007/978-3-642-21643-5_8

Dow, S.P., Heddleston, K. and Klemmer, S.R. (2009), "The Efficacy of Prototyping Under Time Constraints", in: Proceedings of the Seventh ACM Conference on Creativity and Cognition, C\&C '09. ACM, New York, NY, USA, pp. 165-174. https://doi.org/10.1145/1640233.1640260

Eisenhardt, K.M. (1989), "Building theories from case study research". Acad. Manage. Rev. Vol. 14, pp. 532-550. https://doi.org/10/bpvq6s

Eppinger, S. and Ulrich, K. (2011), Product Design and Development, 5th Edition. ed. McGraw/Hill, New York.

Forest, C.R., Moore, R.A., Jariwala, A.S., Fasse, B.B., Linsey, J., Newstetter, W., Ngo, P. and Quintero, C. (2014) “The Invention Studio: A University Maker Space and Culture”. Adv. Eng. Educ. Vol. 4 No. 2.

Gerstenberg, A., Sjöman, H., Reime, T., Abrahamsson, P. and Steinert, M. (2015), “A Simultaneous, Multidisciplinary Development and Design Journey - Reflections on Prototyping”, in: Chorianopoulos, K., Divitini, M., Baalsrud Hauge, J., Jaccheri, L. and Malaka, R. (Eds.), Entertainment Computing - ICEC 2015. Springer International Publishing, Cham, pp. 409-416.

Jensen, M.B., Semb, C.C.S., Vindal, S. and Steinert, M. (2016), "State of the Art of Makerspaces - Success Criteria When Designing Makerspaces for Norwegian Industrial Companies". Procedia CIRP, Vol. 54, pp. 65-70. https://doi.org/10.1016/j.procir.2016.05.069

Kelley, D. and Kelley, T. (2013), Creative confidence: Unleashing the creative potential within us all, 1st ed. Crown Publishing Group, New York.

Koedinger, K.R., Kim, J., Jia, J.Z., McLaughlin, E.A. and Bier, N.L. (2015), "Learning is not a spectator sport: Doing is better than watching for learning from a MOOC", in: Proceedings of the Second (2015) ACM Conference on Learning@ Scale. ACM, pp. 111-120. https://doi.org/10.1145/2724660.2724681

Kriesi, C., Bjelland, Ø. and Steinert, M. (2018), "Fast and iterative prototyping for injection molding-a case study of rapidly prototyping”. Procedia Manuf. Vol. 21, pp. 205-212. https://doi.org/10.1016/j.promfg.2018.02.112

Kriesi, C., Blindheim, J., Bjelland, Ø. and Steinert, M. (2016), "Creating Dynamic Requirements through Iteratively Prototyping Critical Functionalities”. Procedia CIRP, Vol. 50, pp. 790-795. https://doi.org/10.1016/j.procir.2016.04.122

Leifer, L.J. and Steinert, M. (2011), "Dancing with ambiguity: Causality behavior, design thinking, and tripleloop-learning”. Inf. Knowl. Syst. Manag. pp. 151-173. https://doi.org/10.3233/IKS-2012-0191

Nonaka, I., Toyama, R. and Konno, N. (2000), "SECI, Ba and Leadership: a Unified Model of Dynamic Knowledge Creation”. Long Range Plann. Vol. 33, pp. 5-34. https://doi.org/10/fv4sms

Nygaard, T., Kriesi, C., Sjöman, H. and Steinert, M. (2018), "From the Eyes of the Patient - Real Time Gaze Control of Medical Training Mannequins", in: Proceedings of NordiCHI2018. Presented at the NordiCHI 2018, Oslo, NO. https://doi.org/10.1145/3240167.3240228

Rising, L. and Janoff, N.S. (2000), "he Scrum software development process for small teams". IEEE Softw. Vol. 17, pp. 26-32. https://doi.org/10.1109/52.854065

Slåttsveen, K., Kriesi, C., Steinert, M. and Aasland, K.E. (2018), "Experiences from a positivistic Way of teaching in the Fuzzy Front End", in: Proceedings of the 20th International Conference on Engineering and Product Design Education (E\&PDE18), Design Education, London, United Kingdom, 6th \& 7th September 2018. Presented at the International Conference on Engineering and Product Design Education, Design Society, London, pp. 694-699.

Steinert, M. and Leifer, L.J. (2012), "Finding One's Way": Re-Discovering a Hunter-Gatherer Model based on Wayfaring". Int. J. Eng. Educ. Vol. 28, p. 251.

Winjum, J., Wulvik, A., Erichsen, J.A.B., Welo, T. and Steinert, M. (2017), “A Heuristic Approach for EarlyStage Product Development in Extreme Environments". Presented at the International Conference on Engineering, Technology and Innovation (ICE/ITMC), IEEE, Madeira, Portugal, pp. 635-641. https://doi.org/10.1109/ICE.2017.8279942

Yin, R.K. (2013), Case study research: Design and methods, 6th ed. SAGE Publications Inc, Los Angeles.

\section{ACKNOWLEDGEMENTS}

We would like to extend our gratitude to the students involved in the projects presented for letting us observe their process. This research is supported by the Research Council of Norway through its userdriven research (BIA) funding scheme, project number 236739/O30. 